(C) The Author(s), 2021. Published by Cambridge University Press on behalf of The Nutrition Society. This is an Open Access article, distributed under the terms of the Creative Commons Attribution licence (http://creativecommons.org/licenses/by/4.0/), which permits unrestricted re-use, distribution, and reproduction in any medium, provided the original work is properly cited.

\title{
Bone mineral density at age 7 years does not associate with adherence to vitamin $D$ supplementation guidelines in infancy or vitamin $D$ status in pregnancy and childhood: an Odense Child Cohort study
}

\author{
Signe Monrad Nørgaard ${ }^{1,2}$, Christine Dalgård ${ }^{3}$, Malene Søborg Heidemann ${ }^{2,4}$, Anders Jørgen Schou ${ }^{2,4}$ and \\ Henrik Thybo Christesen ${ }^{1,2 *}$ \\ ${ }^{1}$ Department of Clinical Research, Faculty of Health Sciences, University of Southern Denmark, Odense C, Denmark \\ ${ }^{2}$ Hans Christian Andersen Children's Hospital, Odense University Hospital, Odense C, Denmark \\ ${ }^{3}$ IST - Clinical Pharmacology, Pharmacy and Environmental Medicine, University of Southern Denmark, Odense C, Denmark \\ ${ }^{4}$ Steno Diabetes Center Odense, Odense University Hospital, Odense C, Denmark
}

(Submitted 16 July 2020 - Final revision received 7 January 2021 - Accepted 18 January 2021 - First published online 26 January 2021)

Abstract

Vitamin D supplementation in infancy is recommended to prevent rickets. At the population level, its effects on bone mineralisation are largely unknown. We aimed to explore whether adherence to national vitamin D supplementation guidelines (10 $\mu \mathrm{g} / \mathrm{d}$ up to the age of 2 years), supplementation at the ages of 5 and 7 years, and serum 25-hydroxyvitamin D $(\mathrm{s}-25(\mathrm{OH}) \mathrm{D})$ at various time points associated with bone mineral density (BMD) at the age of 7 years in the Odense Child Cohort, Denmark ( $n$ 1194). High adherence was defined as supplementation with $10 \mu \mathrm{g}$ of vitamin D 6-7 times per week during $\geq 80 \%$ of the observation time. s-25(OH)D was analysed using LC-MS/MS. Total-body-less-head (TBLH) BMD was measured by dual-energy X-ray absorptiometry. At the median age of $18 \cdot 1$ months, $53.9 \%(n 475 / 881)$ reported high adherence. The median s-25(OH)D was $64 \cdot 7,78.8,46.0$ and $71.8 \mathrm{nmol} / \mathrm{l}$ in early pregnancy, late pregnancy, cord blood and at 5 years, respectively. The mean TBLH BMD at the median age of 7.1 years was 0.613 (sD 0.049$) \mathrm{g} / \mathrm{cm}^{2}(z$-score +0.363 (sD 0.824)). In adjusted analyses, vitamin D supplementation up to 18 months, and at 5 and 7 years, was not associated with TBLH BMD. Similarly, no robust associations were found between TBLH BMD and s-25(OH)D at any time point. No associations were found for TBLH bone mineral concentration or bone area. In this population with relatively high s-25(OH)D concentrations, no consistent associations were found between adherence to vitamin D supplementation recommendations or vitamin D status in pregnancy or childhood, and bone mineralisation at the age of 7 years.

Key words: Bone mineral density: Vitamin D supplementation: 25-Hydroxyvitamin D: Children: Dual-energy X-ray absorptiometry

Vitamin D and Ca are important for ensuring optimal bone accretion in childhood and thereby maximising peak bone mass, which may prevent osteoporosis and bone fractures later in life ${ }^{(1-4)}$.

In fetal life, bone mineralisation is independent of vitamin D per se, but maternal calcitriol concentration increases during pregnancy, ensuring Ca supply to the fetus ${ }^{(5)}$. According to a recent global consensus report, a daily vitamin D supplementation of $15 \mu \mathrm{g}$ is recommended for all pregnant women to prevent hypocalcaemia, dental enamel malformations and congenital rickets in offspring ${ }^{(6)}$.

Furthermore, all infants should, irrespective of diet, use vitamin $\mathrm{D}$ supplementation $(10 \mu \mathrm{g}$ per day from birth to the age of 12 months) to prevent nutritional rickets. In Denmark, a daily vitamin D supplementation of $10 \mu \mathrm{g}$ has until January 2020 been recommended for pregnant women and all children up to the age of 24 months, regardless of vitamin D intake through diet and fortified formula milk, to support maximal bone growth $^{(7,8)}$. Sun exposure is, through UV-B radiation, an important source for endogenous synthesis of vitamin D and thus a determinant for vitamin D status ${ }^{(9)}$. However, direct sun exposure is not recommended for neonates and infants and should be limited in older children to protect from skin cancer ${ }^{(6,10)}$.

Evidence is conflicting whether vitamin D supplementation in pregnancy improves growth or bone mass accretion in the offspring, although programming theories have been proposed $^{(11-18)}$. In addition, randomised controlled trials (RCT)

Abbreviations: BA, bone area; BMC, bone mineral content; BMD, bone mineral density; DXA, dual-energy X-ray absorptiometry; IQR, interquartile range; OCC, Odense Child Cohort; RCT, randomised controlled trial; s-25(OH)D, serum 25-hydroxyvitamin D; TBLH, total body less head..

* Corresponding author: H. T. Christesen, fax +456591 1862, email henrik.christesen@rsyd.dk 
on the possible effect of vitamin D supplementation in healthy school-aged children on total body or total-body-less-head (TBLH) bone mineral content (BMC) and bone mineral density (BMD) report diverging results ${ }^{(19-22)}$, although the majority of the studies find no significant improvements at follow-up ${ }^{(19,21,22)}$.

In an RCT with vitamin D supplementation doses ranging from 10 to $40 \mu \mathrm{g}$ given daily to children at the age of 1 month until 12 months, the total body BMD and BMC measured by dual-energy X-ray absorptiometry (DXA) scans at 1 and 3 years were similar between the groups ${ }^{(23,24)}$. However, an observational study on breastfed infants found an increase in bone mineralisation in children who used a daily supplementation of $10 \mu \mathrm{g}$ of vitamin $\mathrm{D}$ compared with those who did not use supplementation $^{(25)}$.

According to the Institute of Medicine, serum 25-hydroxyvitamin D (s-25(OH)D) concentrations above $50 \mathrm{nmol} / \mathrm{l}$ are sufficient to ensure optimal bone health in adults ${ }^{(26)}$. Vitamin D supplementation to adults with vitamin D sufficiency may therefore by redundant. However, less is known about this for children. In the Danish population-based Odense Child Cohort (OCC), where vitamin D status in pregnancy and childhood is relatively high, we have previously found that s-25(OH)D measured in early and late pregnancy and in cord blood was neither associated with offspring skull parameters, nor total length or height up to 3 years of age $\mathrm{ag}^{(27,28)}$.

Adherence to vitamin D supplementation recommendations may be far from optimal ${ }^{(29,30)}$, and the associations with bone mineralisation are largely unknown in children. Therefore, we examined the association between adherence to vitamin D supplementation recommendations for children up to at the age of 18 months and DXA TBLH BMD at the age of 7 years. Furthermore, we examined the association between DXA TBLH BMD and s$25(\mathrm{OH}) \mathrm{D}$ measured in early and late pregnancy, umbilical cord and at the age of 5 years. Finally, we examined the associations between vitamin D supplementation use at the ages of 5 and 7 years, and DXA TBLH BMD at the age of 7 years.

\section{Population and methods}

The OCC design and protocol have been described previously ${ }^{31)}$; briefly, all pregnant women ( $n$ 6707) living in Odense Municipality, Denmark, from 2010 through 2012 were invited to participate and 2876 women were enrolled.

Blood samples were collected from mothers in early and late pregnancy (before and after 20 weeks, respectively), in umbilical cord at birth, and from children at 5 years of age. Questionnaires on vitamin D supplementation, diet, physical activity, lifestyle, socio-demographic factors and diseases were collected twice during pregnancy and at 3 months, 18 months, 5 years and 7 years of age. The children were physically examined at the ages of 3 months, 18 months, 5 years and 7 years. To ensure that the children were of the same age at the time of the examinations, they were invited to participate at a date close to their birthday.

In the present study, OCC participants with either available data on vitamin D supplementation at the ages of 18 months, 5 years, or 7 years or s-25(OH)D at any age and available DXA scan at the age of 7 years were included. Exclusion criteria were multiple pregnancy, chronic diseases that may affect bone mineralisation and growth, for example, diabetes, leukaemia and neurodevelopmental diseases, and treatment with systemic corticosteroids for longer than 3 months in total. Children born preterm were not excluded from the study unless being affected by other diseases; instead, we chose to adjust for gestational age at birth to account for potential effects on bone mineralisation.

\section{Assessment of adherence to recommendations on vitamin D supplementation}

Intake of vitamin D supplementation was determined through a questionnaire at child age 18 months. Parents were asked to report whether their child used or had previously used vitamin $\mathrm{D}$ supplementation, and in that case further report duration and frequency. Children were defined as having high adherence if they used $10 \mu \mathrm{g}$ of vitamin D supplementation 6-7 times per week during at least $80 \%$ of the observation time. Non-adherence was defined as all intakes lower than that of the high-adherence group. The non-adherent group was further divided into intermediate adherence, defined as using vitamin $\mathrm{D}$ supplementation for longer than 6 months, but without fulfilling the criteria of the high-adherence group, and low adherence defined as using supplementation for 6 months or less.

\section{Assessment of vitamin D status}

All blood samples were collected using BD Vacutainers serum clot activator tubes, reference 309032 . The blood samples were stored at $-80^{\circ} \mathrm{C}$ until s-25(OH)D was analysed by the LC-MS/MS method as previously described ${ }^{(32)}$. The accuracy of the method was validated using Standard Reference Material 972 'Vitamin D in Human Serum', the National Institute of Standards and Technology. s-25(OH)D $\mathrm{D}_{3}$ was on average (target), for low-, midand high-level QC, 16.7 (16.9) nmol/l, 53.4 (54.7) nmol/1 and 105.5 (107) nmol/l), respectively. The $\mathrm{CV}$ were $8 \cdot 1,5.5$ and $6.6 \%$, respectively. Vitamin $\mathrm{D}_{2}$ was on average (target), for low-, mid- and high-level QC, $16 \cdot 2$ (15.3) nmol/1, $51 \cdot 7$ (49.1) nmol/1 and $103.9(97 \cdot 1) \mathrm{nmol} / \mathrm{l}$. The CV were $6 \cdot 9,5 \cdot 1$ and $6 \cdot 1 \%$, respectively. C3-epimers were included in the s-25(OH)D measurements. In a tested subsample, $\mathrm{C} 3$-epimer concentrations in pregnancy and cord blood were between 1.1 and $3.0 \mathrm{nmol} / 1$, that is, $0.5-2.3 \%$ of total s-25(OH)D concentration ${ }^{(33)}$.

\section{Assessment of bone mineral density}

The primary outcome was TBLH BMD at 7 years of age, obtained by whole-body DXA scans using a Prodigy DXA scanner with enCORE software version 17 (GE Healthcare, General Electric Company). The children were positioned on the back, wearing only underpants, while the scan was performed in one movement from head to toes with an estimated entrance skin dose of $0.4 \mu \mathrm{Gy}$. The biomedical laboratory scientists who performed the scans ensured that the children were positioned correctly and laying still during the scan; if this was not the case, the scan was repeated. The enCORE software automatically adjusted placement of landmarks. All scans were assesed by a biomedical laboratory assistant who checked the positioning and the quality and evaluated if it was nescessary for a paediatrician to also 
assess the scan. The precision and reproducibility of the DXA scanner were tested by daily scans of a phantom and additional controls once per month following the guideline from the manufacturer. The CV was between 0.2 and $0.4 \%$. As the DXA scans were performed as part of the physical examination close to the children's 7-year birthdays, they were performed year round.

\section{Assessment of covariates}

At child age 3 months, skintone was determined at the buttock through examination using the Fitzpatrick scale ${ }^{(34)}$. At the age of 7 years, barefooted height was measured in centimetres with 1-decimal precision using a Seca 213 portable stadiometer (SECA International). Child weight was measured wearing minimal clothing in $\mathrm{kg}$ with 1-decimal precision using Seca 861 digital scales. BMI was calculated by weight in kg divided by height in metres squared. Pubertal stage was determined by physical examination (inspection and palpation) of breast tissue and testicle volume using an orchidometer according to the Tanner scale $^{(35,36)}$. All physical examinations were conducted by trained biomedical laboratory scientists.

Also at child age 7 years, parents were asked to indicate by categories rounded to intergers (1-2 dl, 3-4 dl, 5-6 dl and >6 dl) the amount of dairy products (milk and yogurt) the child consumed daily, using a survey question from the Danish National Birth Cohort. ${ }^{(37)}$ The children were grouped into those consuming at least $3 \mathrm{dl}$ and thus adhered to the recommendations of the Danish Health Authorities $(\geq 2.5 \mathrm{dl})$ and those consuming less. Furthermore, parents were asked to indicate how many days of a normal month the child would eat meat for dinner. Finally, the parents were asked to evaluate how active the child was compared with other children of the same age and sex by selecting one of the following options: 'far less active', 'a little less active', 'equally active', 'a little more active' or 'far more active'. These options were reduced to 'less active', 'equally active' and 'more active'. The question was inspired from the questions used in Sallis et al. ${ }^{(38)}$

Information on the following parameters was validated using medical records: mother's age and parity after delivery, smoking in pregnancy, gestational age at birth, sex, and child body length and weight at birth.

\section{Statistical analyses}

Differences between the high-adherent and non-adherent groups, participants and non-participants, and participants with complete and incomplete covariate data were tested using the two-sample Wilcoxon rank sum (Mann-Whitney) test on non-normally distributed variables, Pearson's $\chi^{2}$ test on categorical variables and the two-sample $t$ test with equal variances on normally distributed variables.

We used multiple linear and logistic regressions to examine the association between adherence to vitamin D supplementation recommendations and BMD at the age of 7 years. In our primary analyses, we used adherence at the age of 18 months as the exposure and TBLH BMD at the age of 7 years as the outcome. As the BMD $z$-score differed from the reference population, we instead used TBLH BMD adjusted for height and child sex as mandatory variables in the basic model 1 . Also, the mandatory variable skin tone was considered for this model as an indicator of ethnicity but was instead included in model 3 due to missing values. Model 2 further adjusted for gestational age, parity and child BMI. Model 3 adjusted for all the above as well as physical activity, skin tone and daily dairy product consumption, as information on these covariates were missing for some of the participants.

The covariates included in the models were chosen a priori based on a review of the literature and their association with either the exposure or outcome. Covariates associated with exposure were gestational age and parity. Alcohol consumption in pregnancy was not included as very few participants consumed alcohol more than once. Smoking, maternal education level and season of medical examinations were not included as they were neither associated with the exposure nor outcome. Covariates univariately associated with the outcome were child height, child weight (BMI), daily intake of dairy products and amount of physical activity compared with peers as estimated by the parents (all measured at the age of 7 years).

We performed the analyses defined a priori stratified by child sex and tested for possible effect modification because sex differences in bone mineralisation have been reported already in childhood ${ }^{(39,40)}$. Furthermore, we examined the association between the use of vitamin D supplementation in pregnancy and at the age of 5 years and s-25(OH)D in early pregnancy, late pregnancy and at the age of 5 years, respectively, by applying multiple linear regression adjusting for the same covariates as in model 3. Finally, we performed multiple logistic regressions with BMD categorised as low (BMD $\leq 10$ th percentile) or normal (BMD $>10$ th percentile) using the same adjustments as in the linear regressions. The same approach was used when analysing the a priori defined secondary exposures, substituting adherence at the age of 18 months with s-25(OH)D concentrations in early and late pregnancy, in umbilical cord, and at the age of 5 years as well as the vitamin D supplementation use at the ages of 5 and 7 years.

When investigating relevant interactions in the models, interactions were found between skin tone and dairy product consumption for vitamin D adherence at the age of 18 months, and between physical activity and BMI for s-25(OH)D at the age of 5 years. Adding these interactions to the models did, however, not change any results (results not shown).

Model assumptions were evaluated for the final model 3 by checking normality and homoscedasticity of residuals, multicollinearity, linearity and model specifications. As none of the participants were siblings, all observations were independent. Child BMI took the place of weight in all regression models, after which no assumptions were violated. Sensitivity analyses were carried out using weight instead of BMI. This did not change any of the results (results not shown).

Two-sided $P$ values $<0.05$ were defined as statistically significant. With a sample size of 881 participants, a power of $80 \%$ and the TBLH BMD standard deviation of $0 \cdot 05$, the minimal detectable difference was $0.09 \mathrm{~g} / \mathrm{cm}^{2}$ between those who adhered to the guidelines and those who did not. Lastly, a backwards multiple linear regression analysis was performed to identify predictors for TBLH BMD. Data were analysed using Stata/IC 16 (StataCorp, LLC). 


\section{Ethical considerations}

The present study was conducted according to the guidelines laid down in the Declaration of Helsinki and all procedures involving human subjects were approved by the Regional Scientific Ethical Committee for Southern Denmark (no. S-20090130) and the Institutional Review Board (no. 18/47377). Written informed consent was obtained from all parents of subjects. Parents were informed about the risks of radiation before DXA scans were conducted. Participation was voluntary for all children and consent could be withdrawn at any time. Data were stored in Odense Patient Data Exploratory Network (OPEN) and in Odense Municipality's Data Warehouse.

\section{Results}

Of the 2876 participants in OCC, 2640 subjects were eligible for participation in the present study. Of these, 1194 participants $(45.2 \%)$ were finally included by having available DXA scans at the age of 7 years and data on at least one of the following parameters: vitamin $\mathrm{D}$ supplementation up to the ages of 18 months, 5 years or 7 years; or s-25(OH)D in early pregnancy, late pregnancy, umbilical cord or at the age of 5 years (Fig. 1). The characteristics of participants are presented in Table 1. The median age when answering the 18-month questionnaire was 18.1 (interquartile range (IQR) 1.3 ) months. The median age at the time of the DXA scan was $7 \cdot 1$ (IQR 0.1) years. All participants were pre-pubertal (Tanner stage 1) and primarily of Western origin. No participants were diagnosed with rickets at any time point.

\section{Adherence to vitamin D supplementation} recommendation up to the age of 18 months

In total, 881 participants had DXA scans and information on vitamin $D$ supplementation use up to the median age of 18.1 (IQR 1.3) months. Of these, 583 (66.2\%) participants used $10 \mu \mathrm{g}$ of vitamin D supplementation 6-7 d per week (Fig. 2(a)), and $626(71.1 \%)$ participants still used vitamin D supplementation at the age of 18 months (Fig. 2(b)). High adherence to the national vitamin $D$ supplementation recommendations was seen in $475 / 881(53.9 \%)$ of the participants (Fig. 2(c)). Only fifty-nine (6.7\%) were in the low-adherence group. Predictors for low or intermediate adherence included higher parity, higher gestational age and alcohol consumption in pregnancy (Table 1). High adherence to vitamin D supplementation recommendation in infancy was positively associated with vitamin $\mathrm{D}$ supplementation use at 5 and 7 years.

\section{Serum 25-hydroxyvitamin D concentrations}

The s-25(OH)D concentrations at the different time points are depicted in Fig. 2(d)-(g). The median s-25(OH)D concentrations were: early pregnancy 64.7 (IQR 28.4$) \mathrm{nmol} / \mathrm{l}$ ( $n$ 600), late pregnancy 78.8 (IQR 35.9) nmol/1 ( $n$ 679), umbilical cord 46.0 (IQR $29 \cdot 7) \mathrm{nmol} / \mathrm{l}(n$ 997) and 5 years of age $71.8(\mathrm{IQR} 32 \cdot 8) \mathrm{nmol} / \mathrm{l}$ ( $n$ 658).

\section{Dual-energy X-ray absorptiometry scan results}

Among our participants ( $n$ 1194), the mean TBLH BMD was 0.613 (SD 0.049) $\mathrm{g} / \mathrm{cm}^{2}$ with a $z$-score of +0.363 (sD 0.824). The median TBLH BMC was 624.748 (IQR 135.255) g. The mean TBLH bone area (BA) was 1029.308 (sD 94.130) $\mathrm{cm}^{2}$.

\section{Associations between vitamin D supplementation and serum 25-hydroxyvitamin D}

In pregnancy and at 5 years, a strong association between vitamin $\mathrm{D}$ supplementation and s-25(OH)D was observed (early pregnancy; $\beta$-coefficient $=9.37(95 \%$ CI $2 \cdot 16,16.59), P=0.01$ late pregnancy; $\beta$-coefficient $=8.90(95 \%$ CI $1.32,16.48), P=0.02$ and 5 years; $\beta$-coefficient 3.79 (95\% CI $2 \cdot 88,4.70), P<0.001)$.

\section{The association between vitamin D supplementation adherence in infancy and dual-energy X-ray absorptiometry scans}

No associations were found between adherence to vitamin D supplementation in infancy and TBLH BMD at the age of 7 years (Table 2). Likewise, adherence to vitamin D supplementation did not associate with TBLH BMD $z$-score, BA or BMC (Table 3). Stratification by sex did not change any of the results (results not shown).

\section{Associations between serum 25-hydroxyvitamin D concentrations and total-body-less-head bone mineral density}

No consistent associations were found between TBLH BMD and $\mathrm{s}-25(\mathrm{OH}) \mathrm{D}$ in early or late pregnancy, in umbilical cord, or at the age of 5 years. These results were consistent using s-25(OH)D on a continuous scale, and when applying either clinical cut-off values or quartiles, as well as when comparing the lowest TBLH BMD 10th percentiles to the remaining values (online Supplementary Tables S1 and S2). As an exception, the odds for having a TBLH BMD $\leq 10$ th percentile was associated with early pregnancy s-25(OH)D $<25 \mathrm{nmol} / 1$ (model 3, adjusted OR $4.82(1 \cdot 08,21 \cdot 57), P=0 \cdot 04, n 16)$ compared with those having a TBLH BMD > 10th percentile (online Supplementary Table S2). Applying the non-interacting covariate season of blood sampling in the models weakened this association to some degree (model 3, adjusted OR 4.35 (0.97, 19.58), $P=0.06, n$ 16). Applying season of blood sampling and/or season of questionnaire answering (data on vitamin D supplementation) did not change any other results at any time point (results not shown).

\section{Recent vitamin D supplementation and total-body-less-head bone mineral density}

Looking at vitamin D supplementation intake at the ages of 5 and 7 years by comparing those using supplementation twice per week or more with those using less, the basic models suggested inverse associations between the use of vitamin D supplementation at the age of 7 years and TBLH BMD, BMC and BMD $z$-score at the age of 7 years. However, these inverse associations disappeared when adjusting for additional variables in model 2 and model 3. At the age of 5 years, no associations were found (online Supplementary Table S3). 


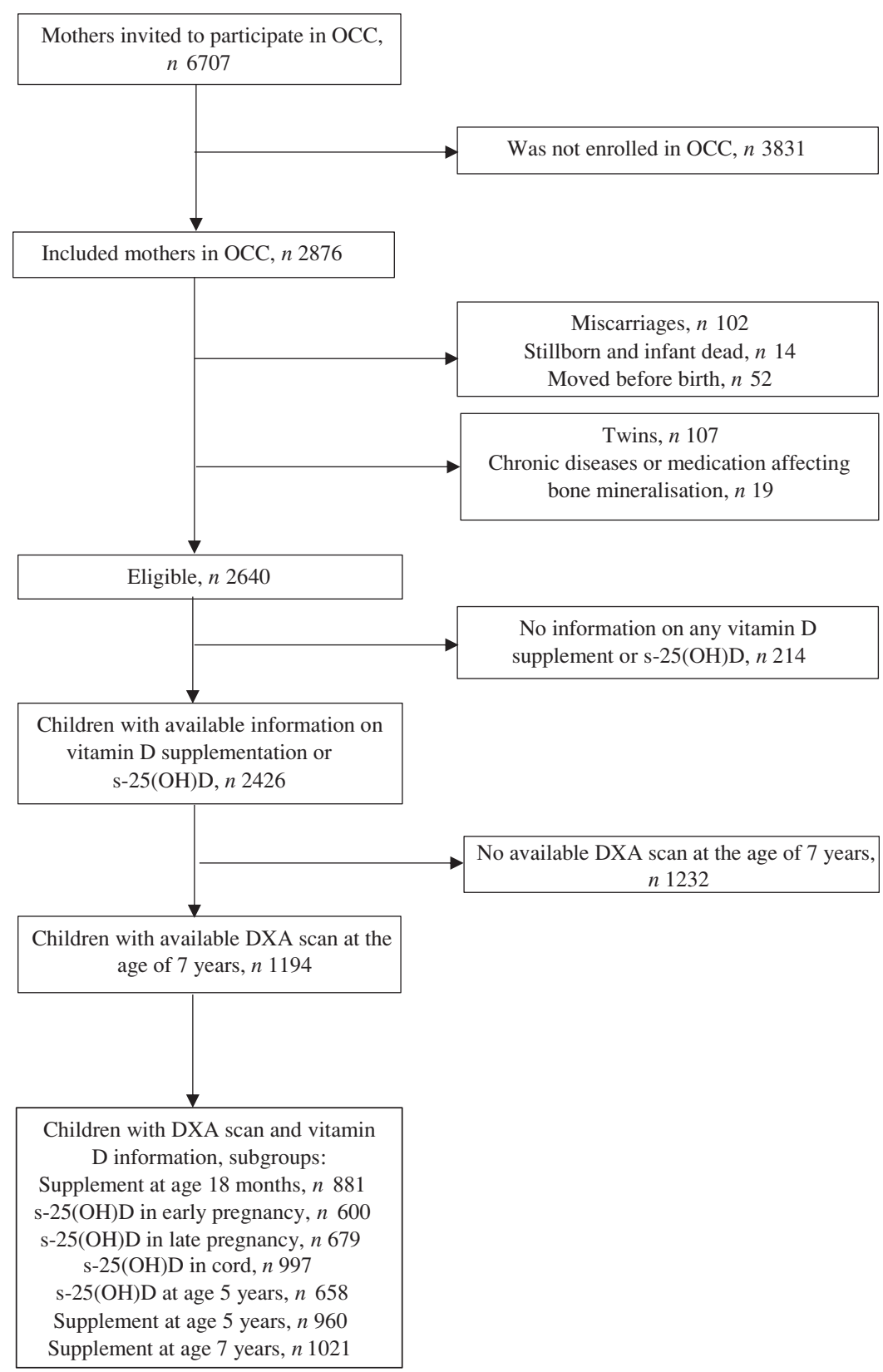

Fig. 1. Participant inclusion flow chart. OCC, Odense Child Cohort; s-25(OH)D, serum 25-hydroxyvitamin D; DXA, dual-energy X-ray absorptiometry.

\section{Other covariates}

In a backwards multiple linear regression analysis, TBLH BMD was positively associated with the current child height, child BMI, physical activity and dairy product consumption. Season at examination, parity, child sex, skin tone and gestational age showed no associations (results not shown).

\section{Differences between participants included in regression} model 1 and model 3

For the main analysis, model 1 included 881 participants. Inclusion of more covariates in model 3 resulted in the exclusion of 144 participants due to missing values. When comparing the 144 excluded participants with the included 737 participants in model 3, no differences were seen for vitamin D supplementation at any age, s-25(OH)D at any age, DXA results or in any other variables included in the models. However, the non-included mothers were younger and more often smoked during pregnancy, had lower educational level, were more likely to have answered the 18-month questionnaire between November and April, and had children who were slightly older at the physical examination at the age of 7 years (online Supplementary Table S4).

\section{Participants and non-participants}

In comparison with our participants, non-participant OCC children had a shorter body length at birth, were exclusively breastfed for shorter time periods and were less likely to have 
NS British Journal of Nutrition

Table 1. Descriptive characteristics of participants in the 18-month adherent and non-adherent groups

(Mean values and standard deviations; numbers and percentages; median values and interquartile ranges (IQR))

\begin{tabular}{|c|c|c|c|c|c|c|c|c|c|c|c|c|c|}
\hline & \multicolumn{4}{|c|}{ Total } & \multicolumn{4}{|c|}{ High adherence } & \multicolumn{4}{|c|}{ Non-adherence } & \multirow[b]{2}{*}{$P \dagger$} \\
\hline & $n$ & $\%$ & Median & IQR & $n$ & $\%$ & Median & IQR & $n$ & $\%$ & Median & IQR & \\
\hline Number of participants & 881 & & & & 475 & 53.9 & & & 406 & $46 \cdot 1$ & & & \\
\hline Mother's age at delivery (years), $n 881$ & & & 31.0 & $6 \cdot 0$ & & & $31 \cdot 0$ & $6 \cdot 0$ & & & $30 \cdot 0$ & $6 \cdot 0$ & 0.36 \\
\hline Mother's parity, $n 881$ & & & & & & & & & & & & & 0.001 \\
\hline & \multicolumn{12}{|c|}{ Maternal education, $n 876$} & \\
\hline Lower & 232 & 26.5 & & & 121 & $25 \cdot 6$ & & & 111 & 27.5 & & & 0.76 \\
\hline Intermediate & 437 & 49.9 & & & 241 & 51.0 & & & 196 & $48 \cdot 6$ & & & \\
\hline Higher & 207 & $23 \cdot 6$ & & & 111 & 23.5 & & & 96 & 23.8 & & & \\
\hline \multicolumn{14}{|l|}{ Smoking in pregnancy, $n 876$} \\
\hline Yes & 38 & $4 \cdot 3$ & & & 18 & 3.8 & & & 20 & $5 \cdot 0$ & & & 0.40 \\
\hline Alcohol in pregnancy, $n 594$ & & & & & & & & & & & & & 0.01 \\
\hline Yes & 74 & 12.5 & & & 30 & $9 \cdot 3$ & & & 44 & $16 \cdot 3$ & & & \\
\hline Vitamin D suppl. in pregnancy§, $n 474$ & & & & & & & & & & & & & 0.05 \\
\hline Yes & 406 & $85 \cdot 6$ & & & 231 & 88.5 & & & 175 & $82 \cdot 2$ & & & \\
\hline Age at DXA scan (years), $n 881$ & & & $7 \cdot 1$ & 0.1 & & & $7 \cdot 1$ & 0.1 & & & $7 \cdot 1$ & 0.1 & 0.41 \\
\hline Sex, $n 881$ & & & & & & & & & & & & & 0.35 \\
\hline Male & 458 & $52 \cdot 0$ & & & 240 & 50.5 & & & 188 & $46 \cdot 3$ & & & \\
\hline Skin tone, Fitzpatrick scale, $n 829$ & & & & & & & & & & & & & 0.53 \\
\hline Fitzpatrick scale $1-2$ & 449 & $54 \cdot 2$ & & & 252 & $55 \cdot 1$ & & & 197 & 53.0 & & & \\
\hline Season at 18 -month questionnaire, $n 881$ & & & & & & & & & & & & & 0.45 \\
\hline November-April & 383 & 43.5 & & & 212 & $44 \cdot 6$ & & & 171 & $42 \cdot 1$ & & & \\
\hline TBLH BMD $\left(\mathrm{g} / \mathrm{cm}^{2}\right), n 881$ & & & & & & & & & & & & & 0.57 \\
\hline Mean & 0.61 & & & & 0.613 & & & & 0.614 & & & & \\
\hline SD & 0.04 & & & & 0.050 & & & & 0.048 & & & & \\
\hline TBLH BMC (g), $n 881$ & & & 624.75 & 134.7 & & & 619.65 & 133.5 & & & 623.22 & 137.1 & 0.57 \\
\hline TBLH BA $\left(\mathrm{cm}^{2}\right), n 881$ & 1028 & 93.80 & & & $1025 \cdot 21$ & 91.48 & & & 1032.07 & 96.42 & & & 0.28 \\
\hline Mean & 0.61 & & & & 0.613 & & & & 0.614 & & & & \\
\hline SD & 0.04 & & & & 0.050 & & & & 0.048 & & & & \\
\hline TBLH $z$-score, $n 881$ & & & & & & & & & & & & & 0.48 \\
\hline Mean & 0.36 & & & & 0.346 & & & & 0.385 & & & & \\
\hline SD & 0.81 & & & & 0.816 & & & & 0.833 & & & & \\
\hline Height (cm), $n 881$ & & & & & & & & & & & & & 0.61 \\
\hline Mean & $125 \cdot 6$ & & & & $125 \cdot 6$ & & & & $125 \cdot 7$ & & & & \\
\hline SD & $5 \cdot 18$ & & & & $5 \cdot 13$ & & & & 5.23 & & & & \\
\hline Weight (kg), $n 881$ & & & $24 \cdot 2$ & 4.7 & & & $24 \cdot 1$ & 4.6 & & & $24 \cdot 0$ & 4.8 & 0.81 \\
\hline Child BMI $\left(\mathrm{kg} / \mathrm{m}^{2}\right), n 881$ & & & $15 \cdot 3$ & 1.9 & & & $15 \cdot 3$ & 1.8 & & & $15 \cdot 4$ & $2 \cdot 0$ & 0.98 \\
\hline Physical activity, $n 791$ & & & & & & & & & & & & & 0.85 \\
\hline Less active & 64 & $8 \cdot 1$ & & & 35 & $8 \cdot 1$ & & & 29 & $8 \cdot 1$ & & & \\
\hline As active & 533 & 67.4 & & & 287 & 66.6 & & & 246 & $68 \cdot 3$ & & & \\
\hline More active & 194 & 24.5 & & & 109 & 24.5 & & & 85 & $23 \cdot 6$ & & & \\
\hline Meat intake (d/month) (7 years), $n 765$ & & & $22 \cdot 0$ & $10 \cdot 0$ & & & $23 \cdot 0$ & $10 \cdot 0$ & & & 21.0 & $11 \cdot 0$ & 0.57 \\
\hline $\begin{array}{l}\text { Daily dairy product consumption (7 years), } n 781 \\
\leq 3 \mathrm{dl} / \mathrm{d}\end{array}$ & 343 & 43.9 & & & 189 & $44 \cdot 1$ & & & 154 & 43.8 & & & 0.93 \\
\hline Gestation age at birth (weeks), $n 879$ & & & $40 \cdot 1$ & 1.7 & & & $40 \cdot 1$ & 1.9 & & & $40 \cdot 3$ & 1.9 & 0.03 \\
\hline Body weight at birth $(\mathrm{g}), n 879$ & & & 3550 & $662 \cdot 0$ & & & 3515 & $640 \cdot 0$ & & & 3580 & $690 \cdot 0$ & 0.20 \\
\hline
\end{tabular}


older siblings. The mothers were younger and had a lower educational level but had higher levels of s-25(OH)D in early pregnancy (online Supplementary Table S5). No difference was seen for adherence to vitamin D supplementation recommendations in infancy.

\section{Discussion}

In this population-based cohort of children and their mothers, no consistent associations were found between vitamin D supplementation use at the ages of 18 months, 5 years and 7 years and TBLH BMD, BMC or BA measured by DXA scan at the age of 7 years. Similarly, no consistent associations were found between s-25(OH)D in early pregnancy, late pregnancy, umbilical cord or at the age of 5 years and TBLH BMD. Our population was representative for the background population cohort with regard to adherence to vitamin D supplementation in infants and the other covariates in the models but differed with regard to age, education level, s-25(OH)D in early pregnancy, and parity of the mothers as well as body length at birth, weeks of exclusive breast-feeding, and holidays of the child and may well differ compared with other populations. For example, the vitamin D status in our study population was generally high, from pregnancy to the age of 5 years. Of note, no participants in the entire OCC had rickets. The value of vitamin D supplementation in pregnancy and infancy to prevent rickets, as confirmed in consensus guidelines ${ }^{(6)}$, was not questioned by our findings.

Our results regarding supplementation in infancy and BMD are compatible with three RCTs ${ }^{(23,24,41,42)}$ investigating the effects of receiving 30 or $40 \mu \mathrm{g}$ of vitamin D supplementation daily in infancy compared with the recommended $10 \mu \mathrm{g}$. None of these studies found significant differences in BMD at follow-up between the ages of 3 months and 3 years. Our study suggests that even lower doses of vitamin D supplementation may be sufficient in a well-off, well-nourished population.

Furthermore, our study suggests that $10 \mu \mathrm{g}$ of daily vitamin D supplementation up to 18 months (compared with less supplementation) has no conditioning effect on BMD later in childhood. A plausible explanation of this finding is that even the low-adherence group in our study may have sufficient s-25(OH)D concentrations due to dietary intake and sun exposure to support an optimal BMD. This is possible because the typical Danish diet is rich in $\mathrm{Ca}^{(43,44)}$, whereby even a lower fractional Ca absorption caused by a suboptimal s-25(OH)D may still result in an overall sufficient Ca uptake ${ }^{(45)}$. Indeed, dairy product consumption was associated with 7-year BMD in the backwards regression. Alternatively, vitamin D supplementation in infancy may temporarily improve bone mineralisation during the first years of life, but without sustained effects.

We did not find any robust associations between s-25(OH)D in pregnancy or cord blood and bone mineralisation. The association between s-25(OH)D $<25 \mathrm{nmol} / 1$ in early pregnancy ( $n$ 16) and TBLH BMD in the lowest 10th percentile weakened in sensitivity analyses after including season in the regression models.

Our general null findings are in line with findings from a large RCT where $25 \mu \mathrm{g}$ of daily supplementation in pregnancy $v$. 
(a) Frequency of supplementation

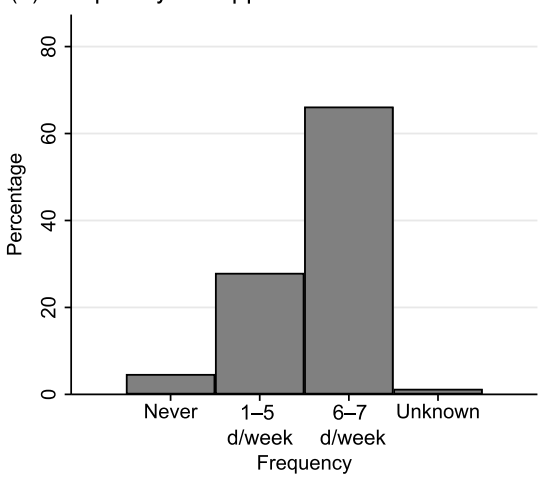

(d) $\mathrm{s}-25(\mathrm{OH}) \mathrm{D}$ in early pregnancy

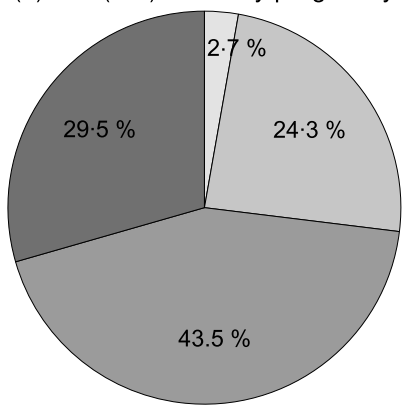

(b) Duration of supplementation

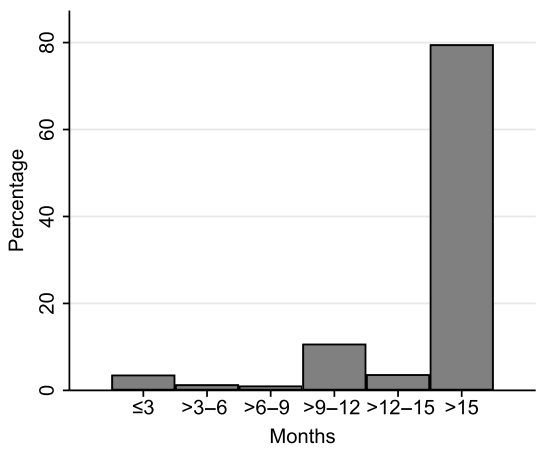

(e) $\mathrm{s}-25(\mathrm{OH}) \mathrm{D}$ in late pregnancy

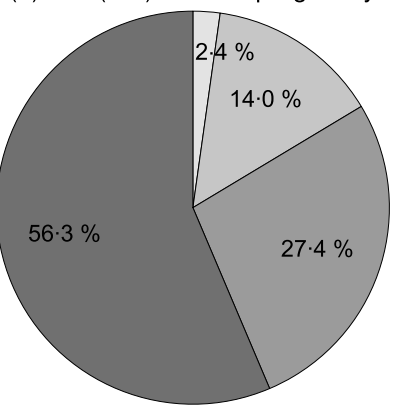

(f) $\mathrm{s}-25(\mathrm{OH}) \mathrm{D}$ in cord blood

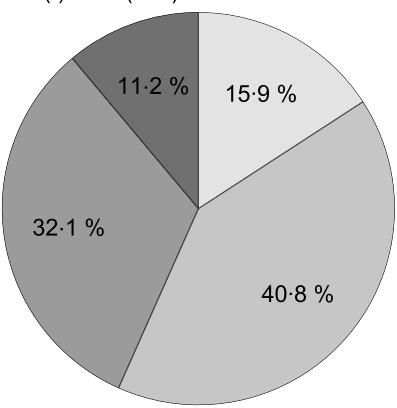

(c) Adherence to supplementation

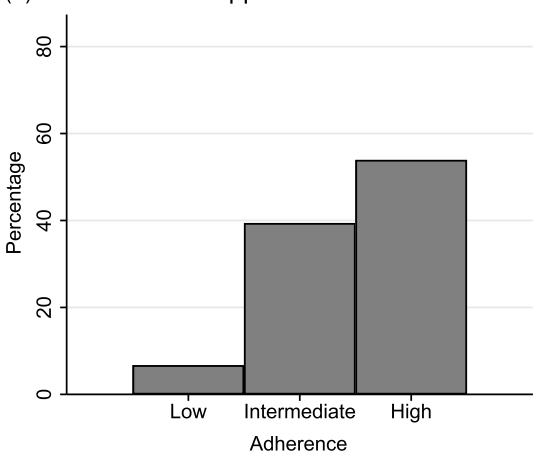

(g) $s-25(\mathrm{OH}) \mathrm{D}$ at the age of 5 years

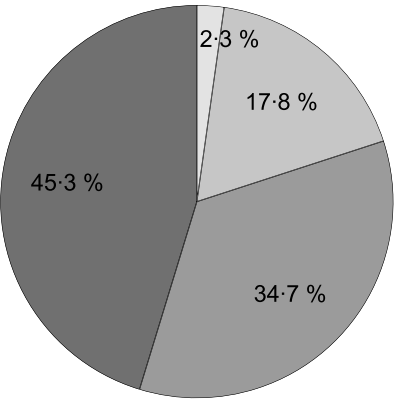

Fig. 2. (a) Frequency and (b) duration of vitamin $D$ supplementation, and (c) adherence to vitamin $D$ supplementation recommendations for children up to the age of 18 months; serum 25-hydroxyvitamin $\mathrm{D}(\mathrm{s}-25(\mathrm{OH}) \mathrm{D}$ ) in (d) early pregnancy (before week 20 of pregnancy), (e) late pregnancy (after week 20 in pregnancy), (f) umbilical cord blood and at $(\mathrm{g})$ the age of 5 years. $\square,<25 \mathrm{mmol} / \mathrm{l} ; \square, 25-50 \mathrm{mmol} / \mathrm{l} ; \square, \geq 50-75 \mathrm{mmol} / \mathrm{l} ; \square,>75 \mathrm{mmol} / \mathrm{l}$.

placebo did not affect newborn total body BMD, BMC and BA ${ }^{(16)}$, and with two large cohort studies observing no associations between s-25(OH)D in pregnancy and offspring bone mineralisation $^{(12,46)}$. Smaller observational studies have observed direct associations ${ }^{(11,47)}$, including the recent Australian cohort study, where $\mathrm{s}-25(\mathrm{OH}) \mathrm{D}<28 \mathrm{nmol} / \mathrm{l}(n \mathrm{12})$ in early pregnancy was associated with TBLH BMD in boys, but not in girls ${ }^{(47)}$. A large observational study ( $n$ 3960), however, failed to detect any associations between s-25(OH)D in any trimester of pregnancy and offspring TBLH BMC at 9.9 years $^{(12)}$. Taken together, the evidence does not support a conditioning role of early or late pregnancy vitamin D status on offspring BMD. This should, however, not be extrapolated to populations with a higher prevalence of vitamin D deficiency.

Brustad et al. ${ }^{(17)}$ randomised pregnant women to different doses of fish oil and to $70 v .10 \mu \mathrm{g}$ of vitamin D daily in the third trimester. In a secondary analysis, a direct effect on TBLH BMC and head BMD by DXA at 3 and 6 years was found, but no difference in the more important outcome TBLH BMD ${ }^{(17)}$. We did not observe any associations for the highest s-25(OH)D concentrations and DXA TBLH BMD, BMC or BA, which, however, does not rule out effects of very high-dose vitamin $\mathrm{D}$ supplementation. Potential adverse effects of high-dose vitamin D supplementation should, however, be taken into account when evaluating recommendations on vitamin D supplementation.

We found no consistent associations between more recent vitamin D supplementation (i.e. at 5 and 7 years) or $25(\mathrm{OH}) \mathrm{D}$ concentrations and TBLH BMD, BMC or BA. This is in line with the results of several other studies performed in the Nordic countries finding no effect of recent vitamin D supplementation on
BMD or BMC in RCTs ${ }^{(21,22)}$, and no associations to recent or current s-25(OH)D in observational studies ${ }^{(48,49)}$. In other countries, results are more diverse $\mathrm{e}^{(19,20,50,51)}$. Both genetic factors as well as dietary intake of $\mathrm{Ca}$, vitamin $\mathrm{D}$, salts, and protein, sun exposure, physical activity, and other lifestyle factors may explain these differences. Our results suggest that recent or current s$25(\mathrm{OH}) \mathrm{D}$ concentrations above $50 \mathrm{nmol} / \mathrm{l}$ are sufficient to support optimal bone health in children, as seen for adults according to the Institute of Medicine ${ }^{(26)}$. For the $20 \%$ of the children with $\mathrm{s}-25(\mathrm{OH}) \mathrm{D}<50 \mathrm{nmol} / 1$ at 5 years, other dietary and lifestyle factors may have compensated to ensure a normal bone mineralisation, as bone mineralisation in children is not dependent of vitamin D status alone, but the combined result of dietary intake of $\mathrm{Ca}$ and vitamin D status ${ }^{(45)}$.

The Danish Health Authorities recommended at the time of the study daily supplementation of $10 \mu \mathrm{g}$ of vitamin $\mathrm{D}$ in pregnancy and from the age of 2 weeks to 2 years to all children. The adherence to the vitamin $\mathrm{D}$ guideline recommendations was high in just above $50 \%$ of participants, but similar or lower adherence has also been detected in recent $\operatorname{Irish}^{(30)}$ and American $^{(29)}$ studies. We observed several well-known risk factors for low adherence to vitamin D supplementation recommendations for children up to the age of 18 months, but also higher parity as a novel risk factor. This is in line with our previous demonstration of higher parity as a risk factor of low vitamin D status in pregnancy ${ }^{(32)}$. Some studies have found higher parity to be associated with lower vitamin D status or vitamin $\mathrm{D}$ intake in pregnancy ${ }^{(52-55)}$, while others have found the contrary ${ }^{(56-58)}$. In our previous OCC studies, higher parity correlated strongly with both lower adherence to vitamin D 
Table 2. Association between adherence to vitamin D supplementation at the age of 18 months and total-body-less-head bone mineral density (TBLH BMD; $\left.\mathrm{g} / \mathrm{cm}^{2}\right)$ at the age of 7 years ${ }^{*} \dagger$

( $\beta$-Coefficients, odd ratios and $95 \%$ confidence intervals)

\begin{tabular}{|c|c|c|c|c|}
\hline TBLH BMD, continuous & Modelł & $\beta$ & $95 \% \mathrm{Cl}$ & $P$ \\
\hline \multirow[t]{3}{*}{ High adherence $v$. non-adherence } & Model 1, $n 881$ & 0.001 & $-0.004,0.006$ & 0.69 \\
\hline & Model 2, $n 881$ & -0.000 & $-0.005,0.005$ & 0.92 \\
\hline & Model 3, n 737 & -0.001 & $-0.006,0.004$ & 0.68 \\
\hline \multirow[t]{3}{*}{ High adherence $v$. low adherence } & Model $1, n 534$ & 0.004 & $-0.007,0.015$ & 0.50 \\
\hline & Model 2, $n 534$ & -0.001 & $-0.012,0.009$ & 0.82 \\
\hline & Model 3, n 454 & 0.003 & $-0.009,0.015$ & 0.65 \\
\hline TBLH BMD, $\leq 10$ th $v .>10$ th percentile§ & Model & OR & $95 \% \mathrm{Cl}$ & $P$ \\
\hline \multirow[t]{3}{*}{ High adherence $v$. non-adherence } & Model 1, $n 881$ & 0.68 & $0.42,1 \cdot 10$ & 0.12 \\
\hline & Model 2, $n 881$ & 0.72 & $0.43,1.20$ & 0.21 \\
\hline & Model $3, n 737$ & 0.80 & $0.45,1.42$ & 0.44 \\
\hline \multirow[t]{3}{*}{ High adherence $v$. low adherence } & Model 1, $n 534$ & 0.80 & $0.31,2.05$ & 0.64 \\
\hline & Model 2, $n 534$ & 1.23 & $0.44,3.45$ & 0.70 \\
\hline & Model $3, n 454$ & 1.91 & $0.60,6.15$ & 0.28 \\
\hline
\end{tabular}

* Differences between the adherence groups were calculated using multiple linear regression for continuous outcomes and multiple logistic regression for percentile outcomes.

† High adherence was defined as consuming $10 \mu \mathrm{g}$ vitamin D supplementation $6-7$ times per week during at least $80 \%$ of the observation time, and non-adherent as otherwise. Low adherence was defined as consuming supplementation for 6 months or less. The reference group is the high-adherence group.

¥ Model 1 is adjusted for height $(\mathrm{cm})$ and sex (male/female). Model 2 is adjusted for height (cm), sex (male/female), gestational age (weeks), parity (unipara/multipara), and child BMI $\left(\mathrm{kg} / \mathrm{m}^{2}\right)$. Model 3 is adjusted for height $(\mathrm{cm})$, sex (male/female), gestational age (weeks), parity (unipara/multipara), child BMI (kg/m²), physical activity (less active than peers/as active as peers/more active than peers), skin tone (Fitzpatrick scale 1-2/3-6), and daily dairy product consumption ( $<3 \mathrm{dl} / \mathrm{d} v$. $\geq 3 \mathrm{dl} / \mathrm{d}$ ).

$\S>10$ th percentile $\left(>0.554 \mathrm{~g} / \mathrm{cm}^{2}\right)$ is the reference group.

Table 3. Association between adherence to vitamin $D$ supplementation at the age of 18 months and bone mineral density (BMD) $z$-score, bone mineral content $(\mathrm{BMC})(\mathrm{g})$, and bone area $(\mathrm{BA})\left(\mathrm{cm}^{2}\right)$ at the age of 7 years*

( $\beta$-Coefficients and $95 \%$ confidence intervals)

\begin{tabular}{|c|c|c|c|c|}
\hline & Model† & $\beta$ & $95 \% \mathrm{Cl}$ & $P$ \\
\hline \multicolumn{5}{|l|}{ TBLH BMD z-score } \\
\hline \multirow[t]{3}{*}{ High adherence $\neq v$. non-adherence } & Model 1, $n 881$ & 0.018 & $-0.069,0.106$ & 0.68 \\
\hline & Model 2, $n 881$ & -0.003 & $-0.084,0.078$ & 0.95 \\
\hline & Model 3, $n 737$ & -0.014 & $-0.100,0.072$ & 0.76 \\
\hline \multirow[t]{3}{*}{ High adherence $v$. low adherence§ } & Model 1, $n 534$ & 0.031 & $-0.155,0.216$ & 0.75 \\
\hline & Model 2, $n 534$ & -0.060 & $-0.235,0.115$ & 0.50 \\
\hline & Model 3, n 454 & 0.007 & $-0.192,0.205$ & 0.95 \\
\hline \multicolumn{5}{|l|}{ TBLH BMC } \\
\hline \multirow{3}{*}{ High adherence $v$. non-adherence } & Model 1, $n 881$ & 3.857 & $-3.370,11.084$ & 0.30 \\
\hline & Model 2, $n 881$ & 2.032 & $-3 \cdot 686,7 \cdot 750$ & 0.49 \\
\hline & Model 3, $n 737$ & 1.001 & $-5 \cdot 171,7 \cdot 173$ & 0.75 \\
\hline \multirow[t]{3}{*}{ High adherence $v$. low adherence } & Model 1, $n 534$ & 9.605 & $-5.592,24.802$ & 0.22 \\
\hline & Model 2, $n 534$ & 0.902 & $-11.543,13.346$ & 0.89 \\
\hline & Model 3, n 454 & 4.133 & $-10 \cdot 176,18 \cdot 441$ & 0.57 \\
\hline \multicolumn{5}{|l|}{ TBLH BA } \\
\hline \multirow[t]{3}{*}{ High adherence $v$. non-adherence } & Model 1, $n 881$ & 4.232 & $-1 \cdot 888,10 \cdot 353$ & 0.18 \\
\hline & Model 2, $n 881$ & 3.453 & $-1.831,8.738$ & 0.20 \\
\hline & Model 3, n 737 & 3.258 & $-2.577,9.092$ & 0.27 \\
\hline \multirow[t]{3}{*}{ High adherence $v$. low adherence } & Model 1, $n 534$ & 9.365 & $-3 \cdot 627,22 \cdot 358$ & 0.18 \\
\hline & Model 2, $n 534$ & 3.729 & $-7.515,14.973$ & 0.52 \\
\hline & Model 3, n 454 & 1.620 & $-11.654,14.894$ & 0.81 \\
\hline
\end{tabular}

TBLH, total-body-less-head.

* Differences between the adherence groups were calculated using multiple linear regression.

† Model 1 is adjusted for height (cm) and sex (male/female). Model 2 is adjusted for height (cm), sex (male/female), gestational age (weeks), parity (unipara/multipara) and child BMI $\left(\mathrm{kg} / \mathrm{m}^{2}\right)$. Model 3 is adjusted for height $(\mathrm{cm})$, sex (male/female), gestational age (weeks), parity (unipara/multipara), child BMl ( $\left.\mathrm{kg} / \mathrm{m}^{2}\right)$, physical activity (less active than peers/as active as peers/more active than peers), skin tone (Fitzpatrick scale 1-2/3-6) and daily dairy product consumption ( $<3 \mathrm{dl} / \mathrm{d} v$. $\geq 3 \mathrm{dl} / \mathrm{d})$.

¥ High adherence was defined as consuming $10 \mu \mathrm{g}$ of vitamin D supplementation 6-7 times per week during at least $80 \%$ of the observation time, and non-adherent as otherwise. $\S$ Low adherence was defined as consuming supplementation for 6 months or less.

supplementation recommendations and with lower s-25(OH)D in pregnancy and cord blood ${ }^{(32,59)}$. These results indicate that the differences in vitamin D status between unipara and multipara may be caused by behavioural or social factors rather than having a biological explanation. We speculate that similar factors, for example, variations in perfectionism and domestic workload, may explain the lower adherence to vitamin D supplementation guidelines in infants with increasing parity. Of note, parity was an independent risk factor, ruling out parity as a proxy for maternal age and educational status.

Despite a relatively low adherence to the vitamin D supplementation recommendations evaluated at 18 months, no 
children had rickets, and no differences were found in TBLD BMD or other bone parameters, or anthropometric measures in our cohort. Furthermore, no differences were seen between children who never used vitamin D supplementation or used supplementation for $\leq 6$ months compared with those still using vitamin D supplement as recommended by 18 months of age. This suggests a robustness of bone health regardless of inferior adherence to vitamin D supplementation in well-nourished children living in developed countries, even at higher latitudes. On the other hand, no inverse associations were found to argue against the national recommendations. To our best knowledge, no existing high-quality data support a praxis of vitamin D supplementation in children beyond 12 months of age, except in risk groups.

\section{Strengths and limitations}

Strengths of the present study include its relatively large size as well as the population-based prospective design; information on vitamin D supplementation repeatedly collected through questionnaires thereby limiting the risk of recall bias; and s-25(OH) D measurements performed by the 'gold standard' method at various times from early pregnancy to the age of 5 years. Additionally, the inclusion of information on lifestyle, such as dairy product consumption, physical activity and physical features such as skin tone, height, sex and BMI strengthens the study. Furthermore, our choice of outcome variables follows the consensus guidelines for interpretation of DXA scans in children ${ }^{(60)}$.

A limitation of the present study is its observational nature including inability to draw conclusions regarding causality of findings and risk of residual confounding. Other limitations include risk of selection bias from the background population and the risk of information bias in questionnaires. Furthermore, data were not available on bone mineralisation in infancy, s-25(OH)D at the age of 7 years, sun exposure and nutritional vitamin $D$ intake. However, vitamin D fortification of food was not common in Denmark at the time studied. S-25(OH)D was not measured in the same child at various seasons; therefore, we cannot ensure that these values are representative for the individual status year round. Lastly, our findings may have limited external validity, especially for other populations with differences in, for example, ethnicity, diet, sun exposure, vitamin D supplementation and prevalence of vitamin D deficiency.

\section{Conclusion}

In the population-based OCC, $53.9 \%$ of the participants were highly adherent to the national guidelines on vitamin D supplementation in infancy. No associations were found between vitamin $D$ supplementation use at the ages of 18 months, 5 years and 7 years and TBLH BMD, BMC or BA measured by DXA scan at the age of 7 years. Similarly, s-25(OH)D in early pregnancy, late pregnancy, umbilical cord or at the age of 5 years was not robustly associated with TBLH BMD. Adverse bone effects of low adherence to vitamin D supplementation guidelines in infancy, or low vitamin $\mathrm{D}$ status in pregnancy and childhood, do not seem to be of major concern in the population studied.

\section{Acknowledgements}

We thank participants and staff in OCC, and the Department of Sports Science and Clinical Biomechanics at the University of Southern Denmark for facilitating the DXA scans.

This work was supported by the Odense University Hospital (S. M. N.).

H. T. C. and S. M. N. designed the study; S. M. N. conducted the research; S. M. N. and C. D. analysed the data; S. M. N. and H. T. C. wrote the main part of the manuscript; C. D., A. J. S. and M. S. H. provided expert input. All authors interpreted the findings and critically reviewed the manuscript. All authors read and approved the final manuscript.

The authors declare that no conflicts of interest exist.

\section{Supplementary material}

To view supplementary material for this article, please visit https://doi.org/10.1017/S0007114521000301

\section{References}

1. Pettifor JM \& Prentice A (2011) The role of vitamin D in paediatric bone health. Best Pract Res Clin Endocrinol Metab 25, 573-584.

2. Levine MA (2012) Assessing bone health in children and adolescents. Indian J Endocrinol Metab 16, S205-S212.

3. Rizzoli R, Bianchi ML, Garabédian M, et al. (2010) Maximizing bone mineral mass gain during growth for the prevention of fractures in the adolescents and the elderly. Bone 46, 294-305.

4. Heaney R, Abrams S, Dawson-Hughes B, et al. (2000) Peak bone mass. Osteoporos Int 11, 985-1009.

5. Kovacs CS (2012) The role of vitamin D in pregnancy and lactation: insights from animal models and clinical studies. Annu Rev Nutr 32, 97-123.

6. Munns CF, Shaw N, Kiely M, et al. (2016) Global consensus recommendations on prevention and management of nutritional rickets. J Clin Endocrinol Metab 101, 394-415.

7. Brot C \& Dars $\varnothing$ P (2010) Forebyggelse, diagnostik og behandling af D-vitaminmangel Baggrundsnotat af 27. maj 2010 (Prevention, Diagnostics, and Treatment of Vitamin-D Deficiency Background Paper on 27 May 2010). Sundhedsstyrelsen, National Board of Health. https://www.sst.dk/-/media/Udgivelser/2010/ Forebyggelse_-diagnostik-og-bahndling-af-D-vitaminmangel-bagg rundsnotat.ashx?la=da\&hash=62D44B54D20FCD99A396F30171E D9B90B9FFE6C5.

8. Turck D, Bresson J, Burlingame B, et al. (2018) Update of the tolerable upper intake level for vitamin D for infants. EFSA J 16, 1-118.

9. Kimlin M, Sun J, Sinclair C, et al. (2015) Are the current Australian sun exposure guidelines effective in maintaining adequate levels of 25-hydroxyvitamin D? J Steroid Biochem Mol Biol 155, 264-270.

10. Commitee on Environmental Health American Academy of Pediatrics (1999) Ultraviolet light: a hazard to children. Pediatrics 104, 2.

11. Javaid MK, Crozier SR, Harvey NC, et al. (2006) Maternal vitamin D status during pregnancy and childhood bone mass at age 9 years: a longitudinal study. Lancet 367, 36-43.

12. Lawlor DA, Wills AK, Fraser A, et al. (2013) Association of maternal vitamin D status during pregnancy with bone-mineral content in off spring: a prospective cohort study. Lancet $\mathbf{3 8 1}$, 2176-2183. 
13. Harvey NC, Holroyd C, Ntani G, et al. (2014) Vitamin D supplementation in pregnancy: a systematic review. Health Technol Assess 18, 1-190.

14. Curtis EM, Moon RJ, Dennison EM, et al. (2014) Prenatal calcium and vitamin D intake, and bone mass in later life. Curr Osteoporos Rep 12, 194-204.

15. Zhu K, Whitehouse AJO, Hart PH, et al. (2014) Maternal vitamin D status during pregnancy and bone cohort study. JBone Miner Res 29, 1088-1095.

16. Cooper C, Harvey NC, Bishop NJ, et al. (2016) Maternal gestational vitamin D supplementation and offspring bone health (MAVIDOS): a multi-centre, double-blind, randomised placebocontrolled trial. Lancet Diabetes Endocrinol 4, 393-402.

17. Brustad N, Garland J, Thorsen J, et al. (2020) Effect of high-dose vs standard-dose vitamin D supplementation in pregnancy on bone mineralization in offspring until age 6 years a prespecified secondary analysis of a double-blinded, randomized clinical trial. JAMA Pediatr 174, 419-427.

18. Balasuriya CND, Larose TL, Mosti MP, et al. (2019) Maternal serum retinol, $25(\mathrm{OH}) \mathrm{D}, 1,25(\mathrm{OH})_{2} \mathrm{D}$ concentrations during pregnancy, peak bone mass, trabecular bone score in adult offspring at 26-year follow-up. PLOS ONE 14, e0222712.

19. Fuleihan GE, Nabulsi $M$, Tamim $H$, et al. (2006) Effect of vitamin D replacement on musculoskeletal parameters in school children : a randomized controlled trial. J Clin Endocrinol Metab 91, 405-412.

20. Du X, Zhu K, Trube A, et al. (2004) School-milk intervention trial enhances growth, bone mineral accretion in Chinese girls aged 10-12 years in Beijing. Br J Nutr 92, 159-168.

21. Mølgaard C, Larnkjær A, Cashman KD, et al. (2010) Does vitamin D supplementation of healthy Danish Caucasian girls affect bone turnover and bone mineralization? Bone 46, 432-439.

22. Cheng S, Lyytikäinen A, Kröger H, et al. (2005) Effects of calcium, dairy product, and vitamin D supplementation on bone mass accrual and body composition in 10-12-y-old girls: a 2 -y randomized trial. Am J Clin Nutr 82, 1115-1126.

23. Gallo S, Comeau K, Vanstone C, et al. (2013) Effect of different dosages of oral vitamin D supplementation on vitamin D status in healthy, breastfed infants. JAMA 309, 1785-1792.

24. Gallo S, Hazell T, Vanstone CA, et al. (2016) Vitamin D supplementation in breastfed infants from Montréal, Canada: 25-hydroxyvitamin D and bone health effects from a followup study at 3 years of age. Osteoporos Int 27, 2459-2466.

25. Savino F, Viola S, Tarasco V, et al. (2011) Bone mineral status in breast-fed infants: influence of vitamin D supplementation. Eur J Clin Nutr 65, 335-339.

26. Rosen CJ, Abrams SA, Aloia JF, et al. (2012) IOM committee members respond to endocrine society vitamin $\mathrm{D}$ guideline. J Clin Endocrinol Metab 97, 1146-1152.

27. Egge S, Christensen N, Lykkedegn S, et al. (2018) Cord serum 25-hydroxyvitamin D is not associated with cranial anthropometrics in infants up to 6 months of age. An Odense Child Cohort study. J Bone Miner Metab 36, 700-709.

28. Christensen ME, Beck-Nielsen SS, Dalgård C, et al. (2018) A novel inverse association between cord 25-hydroxyvitamin D and leg length in boys up to three years. An Odense Child Cohort study. PLOS ONE 13, e0198724.

29. Perrine CG, Sharma AJ, Jefferds MED, et al. (2010) Adherence to vitamin D recommendations among US infants. Pediatrics $\mathbf{1 2 5}$, 627-632.

30. Hemmingway A, Fisher D, Berkery T, et al. (2020) Adherence to the infant vitamin D supplementation policy in Ireland. EurJ Nutr (epublication 17 July 2020).

31. Kyhl HB, Jensen TK, Barington T, et al. (2015) The Odense Child Cohort: aims, design, and cohort profile. Paediatr Perinat Epidemiol 29, 250-258.
32. Andersen LB, Abrahamsen B, Dalgård C, et al. (2013) Parity and tanned white skin as novel predictors of vitamin D status in early pregnancy: a population-based cohort study. Clin Endocrinol 79, 333-341.

33. Mydtskov ND, Lykkedegn S, Fruekilde PBN, et al. (2017) S-25hydroxyvitamin D and C3-epimers in pregnancy and infancy: an Odense Child Cohort study. Clin Biochem 50, 988-996.

34. Fitzpatrick TB (1988) The validity and practicality of sunreactive skin types I through VI. Arch Dermatol 124, 869-871.

35. Marshall WA \& Tanner JM (1969) Variations in pattern of pubertal changes in girls. Arch Dis Child 44, 291-303.

36. Marshall WA \& Tanner JM (1970) Variations in pattern of pubertal changes in boys. Arch Dis Child 45, 13-23.

37. Morgen CS, Ängquist L, Baker JL, et al. (2018) Breastfeeding and complementary feeding in relation to body mass index and overweight at ages 7 and $11 \mathrm{y}$ : a path analysis within the Danish National Birth Cohort. Am J Clin Nutr 107, 313-322.

38. Sallis JF, Taylor WC, Dowda M, et al. (2002) Correlates of vigorous physical activity for children in grades 1 through 12: comparing parent-reported, objectively measured physical activity. Pediatr Exer Sci 14, 30-44.

39. Hasselstrøm H, Karlsson KM, Hansen SE, et al. (2006) Sex differences in bone size and bone mineral density exist before puberty. The Copenhagen School Child Intervention Study (CoSCIS). Calcif Tissue Int 79, 7-14.

40. Crabtree NJ, Shaw NJ, Bishop NJ, et al. (2017) Amalgamated reference data for size-adjusted bone densitometry measurements in 3598 children and young adults - The Alphabet Study. J Bone Miner Res 32, 172-180.

41. Rosendahl J, Valkama S, Holmlund-Suila E, et al. (2018) Effect of higher vs standard dosage of vitamin $\mathrm{D}_{3}$ supplementation on bone strength and infection in healthy infants: a randomized clinical trial. JAMA Pediatr 172, 646-654.

42. Holmlund-Suila E, Viljakainen H, Hytinantti T, et al. (2012) High-dose vitamin D intervention in infants-effects on vitamin D status, calcium homeostasis, and bone strength. J Clin Endocrinol Metab 97, 4139-4147.

43. Trolle E, Gondolf UH, Ege M, et al. (2013) Danskernes kostvaner Spaed- og småbørm 2006-2007 (Dietary Habits in Denmark Infants, Young Children 2006-2007), 1st ed. Søborg: DTU Fødevareinstituttet, Danmarks Tekniske Universitet.

44. Pedersen AN, Christensen T, Matthiessen J, et al. (2015) Danskernes kostvaner 2011-2013 (Dietary Habits in Denmark 2011-2013). Søborg: DTU Fødevareinstituttet, Danmarks Tekniske Universitet.

45. Högler W (2015) Complications of vitamin D deficiency from the foetus to the infant: one cause, one prevention, but who's responsibility? Best Pract Res: Clin Endocrinol Metab 29, 385-398.

46. Garcia AH, Erler NS, Jaddoe VW, et al. (2017) 25-Hydroxyvitamin D concentrations during fetal life and bone health in children aged 6 years: a population-based prospective cohort study. Lancet Diabetes Endocrinol 5, 367-376.

47. Hyde NK, Brennan-Olsen SL, Mohebbi M, et al. (2019) Maternal vitamin D in pregnancy and offspring bone measures in childhood: the vitamin D in pregnancy study. Bone 124, 126-131.

48. Hauksson HH, Hrafnkelsson H, Magnusson KT, et al. (2016) Vitamin D status of Icelandic children and its influence on bone accrual. J Bone Miner Metab 34, 580-586.

49. Videhult FK, Öhlund I, Hernell O, et al. (2016) Body mass but not vitamin D status is associated with bone mineral content and density in young school children in northern Sweden. Food Nutr Res 60, 1-8

50. Moyer-Mileur LJ, Xie B, Ball SD, et al. (2003) Bone mass and density response to a 12 -month trial of calcium and vitamin 
D supplement in preadolescent girls. J Musculoskelet Neuronal Interact 3, 63-70.

51. Li J, Ding W, Cao J, et al. (2018) Serum 25-hydroxyvitamin D and bone mineral density among children and adolescents in a Northwest Chinese city. Bone 116, 28-34.

52. Jensen CB, Petersen SB, Granström C, et al. (2012) Sources and determinants of vitamin $\mathrm{D}$ intake in Danish pregnant women. Nutrients 4, 259-272.

53. Gannagé-Yared MH, Chemali R, Yaacoub N, et al. (2000) Hypovitaminosis D in a sunny country: relation to lifestyle and bone markers. J Bone Miner Res 15, 1856-1862.

54. Al-Musharaf S, Fouda MA, Turkestani IZ, et al. (2018) Vitamin D deficiency prevalence and predictors in early pregnancy among Arab women. Nutrients 10, 1-12.

55. Camargo CA, Rifas-Shiman SL, Litonjua AA, et al. (2007) Maternal intake of vitamin D during pregnancy and risk of recurrent wheeze in children at 3 y of age. Am J Clin Nutr 85, 788-795.
56. Shen Y, Pu L, Si S, et al. (2019) Vitamin D nutrient status during pregnancy and its influencing factors. Clin Nutr 39, 1432-1439.

57. Baek JC, Jo JY, Lee SM, et al. (2019) Differences in 25-hydroxy vitamin $\mathrm{D}$ and vitamin $\mathrm{D}$-binding protein concentrations according to the severity of endometriosis. Clin Exp Reprod Med 46, 125-131.

58. Pérez-López FR, Fernández-Alonso AM, Ferrando-Marco P, et al. (2011) First trimester serum 25-hydroxyvitamin D status and factors related to lower levels in gravids living in the Spanish Mediterranean coast. Reprod Sci 18, 730-736.

59. Lykkedegn S, Beck-Nielsen SS, Sorensen GL, et al. (2017) Vitamin D supplementation, cord 25-hydroxyvitamin D and birth weight: findings from the Odense Child Cohort. Clin Nutr 36, 1621-1627.

60. Crabtree NJ, Arabi A, Bachrach LK, et al. (2014) Dual-energy X-ray absorptiometry interpretation and reporting in children and adolescents: the revised 2013 ISCD pediatric official positions. J Clin Densitom 17, 225-242. 